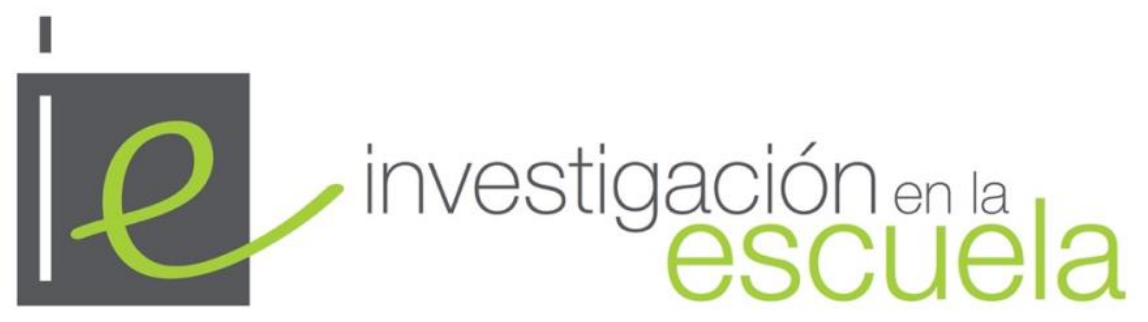

Revista de Investigación e Innovación Educativa nº 102, 2020 | e-ISSN 2443-9991

\title{
¿Puede influir una práctica docente emocional en el cambio de emociones del alumnado? Un estudio en la formación inicial de maestios
}

Can an emotional teaching practice influence the change of emotions of students? A study an initial teacher training

iD Diego Armando Retana Alvarado es Profesor en la Facultad de Educación de la Universidad de Costa Rica (Costa Rica)·diegoarmando.retana@ucr.ac.cr·http://orcid.org/0000-0002-9404-2070

María Ángeles de las Heras Pérez es Profesora Titular en la Facultad de Educación, Psicología y Ciencias del Deporte de la Universidad de Huelva (España)·angeles.delasheras@ddcc.uhu.es · https://orcid.org/0000- 0002$3640-8337$

iD Roque Jiménez Pérez es Catedrático en la Facultad de Educación, Psicología y Ciencias del Deporte de la Universidad de Huelva (España)·rjimenez@ddcc.uhu.es·https://orcid.org/0000-0001-7779-0061

Cómo citar este artículo

Retana-Alvarado, D. A., de las Heras Pérez, M. A. y Jiménez-Pérez, R. (2020). ¿Puede influir una práctica docente emocional en el cambio de emociones del alumnado? Un estudio en la formación inicial de maestros. Investigación en la Escuela, 102, 16-31. doi: http://doi.org/10.12795/IE.2020.i102.02

Resumen. Las emociones son el factor afectivo más influyente en la identidad profesional del profesorado. Las emociones configuran su Conocimiento Didáctico del Contenido (CDC) en la integración de la reflexión y práctica. Este artículo presenta el análisis del cambio en las emociones de 54 maestros en formación inicial hacia una formadora experta, mientras participan en una asignatura de Didáctica de las Ciencias Experimentales con una intervención basada en investigación escolar que promovía la regulación emocional. La intensidad de catorce emociones se analizó a partir del grado de acuerdo en una escala Likert, antes, durante y después de la intervención. También, se llevaron a cabo entrevistas a la formadora y una profesora novel, observaciones de aula y narrativas de los estudiantes para identificar las causas del cambio. Los datos se sometieron a análisis descriptivo, inferencial y de contenido. Los resultados sugieren una mejora en las emociones positivas y una disminución considerable de las emociones negativas. Se detecta un incremento significativo para entusiasmo, satisfacción, interés, tranquilidad, bienestar y asombro.También, se reporta una disminución significativa para temor, frustración, aburrimiento y rechazo. El cambio se relaciona con el perfil vocacional y afectivo de la formadora, quien promueve relaciones auténticas con los futuros maestros a través de su labor con aproximación al modelo de CDC de Gess-Newsome, transfiriendo emociones positivas en el clima de aula.

Abstract. Emotions are the most influential affective factor in the professional identity of teachers. Emotions configure your Pedagogical Content Knowledge (PCK) in the integration of reflection and practice. This article presents the analysis of the change in emotions of 54 teachers in initial training towards an expert trainer, while they participate in a subject of Didactics of Experimental Science with an intervention based on Inquiry Based-Science Education that promoted emotional regulation. The intensity of the emotions was analyzed from the degree of agreement on a Likert scale, before, during and after the intervention. Also, interviews with the trainer and a new teacher, classroom observations and narratives of the students were carried out to identify the causes of change. The data was subject to descriptive, inferential, and content analysis. The results suggest an improvement in positive emotions and a considerable decrease in negative emotions. A significant increase is detected for enthusiasm, satisfaction, interest, tranquility, well-being, and wonder. Also, a significant decrease is reported for fear, frustration, boredom, and rejection. The change is related to the vocational and affective profile of the trainer, who promotes 
authentic relationships with future teachers through her labor with an approximation to the PCK model of GessNewsome, transferring positive emotions in the classroom climate.

Palabras clave $\cdot$ Keywords

Emociones, conocimiento didáctico del contenido, indagación, maestros en formación inicial, Didáctica de las Ciencias Experimentales.

Emotions, pedagogical content knowledge, inquiry, initial teachers training, Didactics of Experimental Sciences.

\section{Introducción}

La enseñanza es una acción ligada al estado emocional en la que se establecen relaciones entre profesores y estudiantes, que implican un esfuerzo intenso para regular las emociones (Frenzel et al., 2016) y crear ambientes de aprendizaje inspiradores donde existan oportunidades para la expresión emocional (Zembylas, 2003). Las emociones son el factor de mayor influencia en la enseñanza y el desarrollo profesional porque asumen un rol central en la toma de decisiones y la formación de la identidad profesional (Shapiro, 2010). Además, sostenemos que los profesores expertos también se distinguen de los principiantes por las emociones que proyectan y gestionan en las buenas prácticas que actúan como referencia en el diseño de intervenciones para la mejora de los afectos y sus manifestaciones.

Desde una perspectiva sociológica y psicosocial, la enseñanza como práctica emocional afecta a los sentimientos y las acciones de otros con quienes los docentes interactúan, que envuelve la propia comprensión de las emociones, y es una forma de labor emocional que para muchos es una tarea de amor. En el profesorado las emociones están conformadas por sus propósitos morales y habilidad (Hargreaves, 1998). Este autor plantea que la enseñanza aparte de implicar sensibilidad emocional también requiere activar la labor emocional, definida por Morris y Feldman (1996) como "el esfuerzo, planeamiento y control necesario para expresar emociones de forma organizada durante las relaciones interpersonales” (p. 987), eso implica que en profesiones como la de docente se espere emociones positivas con carácter altruista, dado que es una acción social que va más allá de la satisfacción de las necesidades propias del profesor.

Hagenauer et al. (2015) refieren que la capacidad de los profesores para gestionar el aula y formar relaciones interpersonales positivas con los estudiantes son factores importantes para su propio bienestar. Esas interacciones pueden evocar emociones positivas y negativas (Sutton y Wheatley, 2003). Los docentes experimentan satisfacción, entusiasmo, alegría, felicidad y orgullo si las relaciones con sus estudiantes son adecuadas o si se cumple un objetivo de enseñanza (Hargreaves, 2000; Schwarzer y Schreiber-Neumann, 1990; Sutton y Harper, 2009). Por el contrario, sienten enfado, frustración y ansiedad cuando el alumnado muestra falta de disciplina y desafía la competencia (Chang y Davis, 2009).

De acuerdo con Sutton y Harper (2009), los profesores experimentados construyen ambientes de aprendizaje productivos a través del humor y la expresión de las emociones positivas. Además, para la reducción de las emociones negativas, emplean estrategias como declaraciones de autoafirmación, que consiste en tener pensamientos positivos; la utilización de un diario de enseñanza emocional, con el propósito de identificar situaciones de emociones intensas y prevenirlas. También estos autores señalan otras estrategias centradas en las respuestas emocionales que modulan la experiencia mediante la respiración profunda (contar hasta diez), el cambio de los pensamientos vinculados con emociones negativas intensas, la relajación, el ejercicio y la planeación preventiva para el día siguiente. En cambio, otros docentes involucran a los estudiantes en discusiones sobre estrategias de regulación emocional, conversan con sus colegas y reflexionan reevaluando los episodios emocionales de la clase.

Dadas las condiciones que anteceden, se reconoce que las emociones del profesorado producen cambios en el Conocimiento Didáctico del Contenido (CDC, en adelante) (Garritz et al., 2015; Melo et al., 2017) cuyos orígenes se asientan en los antecedentes escolares, se inicia en la formación inicial y se desarrolla en la experiencia profesional (Van Driel et al., 2014). Gess-Newsome (2015) propone un modelo de conocimiento profesional que posiciona al CDC desde su naturaleza integrada, precisamente es en la práctica de aula donde interacciona con el contexto y el curriculum. El CDC es un constructo independiente del conocimiento pedagógico y del conocimiento del contenido, aunque relacionado (Kirschner et al., 2016) que sitúa el conocimiento del profesor y la práctica dentro de la enseñanza de una disciplina específica de ciencias (Gess-Newsome et al., 2017). Un modelo más refinado incluye tres esferas de CDC: colectivo, personal y acción (Carlson y Daehler, 2019). Para efectos de esta investigación, el modelo de Gess-Newsome (2015) actúa como marco de referencia en el análisis de la interacción emocional que propicia el cambio.

El estudio se centra en analizar el cambio en la intensidad de las emociones que experimentan futuros maestros hacia una formadora de Didáctica de las Ciencias; en el transcurso de una intervención basada en 
la investigación sobre una cuestión sociocientífica que se acompaña de estrategias autorregulatorias para la gestión emocional, movilizadas por la formadora de manera integrada con los tópicos científicos. Al respecto, hipotetizamos que la propia educación emocional de la formadora contribuye en la transmisión de seguridad, disfrute y tranquilidad, generando cercanía y relaciones horizontales con los futuros maestros, indicadores de una labor emocional. En este artículo se busca responder los siguientes problemas principales:

1. ¿Qué emociones positivas y negativas se manifiestan hacia la formadora?

2. ¿Qué variaciones se detectan en función del momento de la intervención?

3. ¿Cuáles son las posibles causas que facilitan el cambio?

\section{Metodología}

\subsection{Caracterización de la investigación}

Se concibe el fenómeno desde una perspectiva de complejidad que considera el cambio afectivo como faro de luz que se proyecta hacia horizontes de reflexión crítica, colaboración y transformación en las prácticas educativas de formación del profesorado, donde el CDC actúa como pilar básico para la planificación y puesta a punto de la intervención.

De esta manera, el cambio e interacción de las emociones se aborda desde la investigación-acción por el rol activo que asumen los participantes en los problemas que surgen de la práctica. Asimismo, se caracteriza por la necesidad de implicación grupal en la reflexión y toma de decisiones para la transformación del medio social (Rodríguez et al., 1996). En la línea del desarrollo profesional del profesorado de Ciencias, VázquezBernal et al. (2008) sostienen que un programa de investigación-acción focalizado en la innovación y reflexión orientada a la práctica facilita la incorporación gradual de nuevas teorías prácticas a la reflexión y al trabajo en el aula, cuyas acciones están encauzadas mediante el CDC.

Concebimos este trabajo como una investigación con los maestros de Educación Primaria en formación inicial, pues destaca la posibilidad de reflexionar sobre el qué se hace, en un proceso formativo que sienta sus bases en la vinculación de los binomios teoría y práctica, experiencia y reflexión que serán de provecho para su desarrollo personal y profesional (Imbernón, 2012).

Para ello, se enfoca a través de la combinación de diseños de naturaleza cuantitativa y cualitativa porque aporta diversos puntos de vista y métodos que se complementan por medio de la triangulación para alcanzar la comprensión holística de la realidad. Al respecto, Greene (2008) señala que los métodos mixtos son una orientación hacia la investigación social que invita a participar en el diálogo sobre múltiples maneras de dar sentido al mundo. En lo que refiere a la investigación en educación científica, los métodos mixtos a menudo implican una intervención y su evaluación (Treagust et al., 2014).

En este sentido, se concibe el aula como sistema social complejo que potencia la dimensión personal del profesorado para reflejarse en la esfera de su desarrollo profesional. Así, el desarrollo del profesor es condicionado por la interrelación de la gestión emocional, el conocimiento profesional y la investigaciónacción que sustentan el desarrollo personal, profesional y social respectivamente (Bell y Gilbert, 1994; Vázquez-Bernal et al., 2013). Desde la óptica de la Hipótesis de la Complejidad (Vázquez-Bernal et al., 2007, 2012), estas esferas se circunscriben en la complejidad de la reflexión que realiza el profesor cuando planifica la enseñanza, es decir, la reflexión representa categorías que constituyen el CDC personal. Se integra con la complejidad en la práctica que está determinada por el CDC personal y la habilidad en la enseñanza.

\subsection{Contexto formativo y participantes}

La investigación se realiza en el contexto natural de formación docente, específicamente, en el Grado de Maestro en Educación Primaria de la Universidad de Huelva, España. La asignatura Didáctica de Ciencias de la Naturaleza I donde se desarrolló el estudio es de carácter obligatorio y se ubica en el quinto cuatrimestre correspondiente al tercer curso del plan de estudios. Está adscrita al Departamento de Didácticas Integradas, adaptada al Espacio Europeo de Educación Superior y constituida por 6 créditos que comprende 150 horas de trabajo (45 horas lectivas). Se abordan los contenidos científicos escolares de Biología y las competencias emocionales de manera práctica e integrada para que los futuros maestros experimenten bienestar en su dimensión personal y social, a su vez, alcancen madurez emocional mientras desarrollan el CDC.

La profesora, quien impartió la docencia en el grupo T1 donde se llevó a cabo la investigación, es una formadora experta Titular en Didáctica de las Ciencias Experimentales con formación doctoral en Botánica, con diez años de experiencia en dicha asignatura. Su interés en la educación emocional le ha permitido formarse e investigar sobre los aspectos afectivos, habiendo dirigido diversos proyectos de investigación y 
tesis doctorales. En cuanto a la profesora novel, posee formación doctoral en Bioquímica y experiencia como instructora de laboratorios de química.

En términos incidentales, se contó con la participación de 54 estudiantes, con media de edad de 22,16 años $(\mathrm{DT}=4,10)$; procedentes de Huelva, Sevilla, Cádiz, Italia y Alemania. El 89\% realizó estudios de Bachillerato, resultando mayor representación en modalidades de Ciencias Sociales y Humanidades (71\%).

\subsection{Intervención}

El diagnóstico de las emociones al comienzo de la asignatura permitió el diseño, planificación e implementación de una intervención basada en investigación escolar (Cañal et al., 2005), para trabajar el contenido científico ecosistema a partir de la gestión de las emociones, contextualizándolo en la legislación educativa vigente, la cotidianidad local y las aportaciones de otras investigaciones.

La unidad didáctica denominada "Desentrañando secretos controvertidos en el Paraje Natural Los Enebrales de Punta Umbría" aborda la cuestión sociocientífica sobre la construcción de hoteles en un ecosistema sensible a factores antrópicos, específicamente ubicado en el municipio de Punta Umbría, Huelva. Este paraje conserva comunidades de enebros marítimos (Juniperus oxycedrus), conífera endémica de las costas del Atlántico y Mediterráneo, declarada como especie amenazada, debido a la tala indiscriminada y la explotación de sus gálbulos utilizados para la elaboración de ginebra. El ecosistema se caracteriza por la presencia de dunas embrionarias y secundarias, bosque mixto de enebros y sabinas, así como una densa cobertura vegetal de pinos piñoneros. De esta forma, implica la observación e identificación de las características y componentes del ecosistema (Ministerio de Educación, Cultura y Deporte, 2014) y supone la identificación de los factores que inciden en el equilibrio.

Para secuenciar las actividades se sigue un ciclo de aprendizaje constituido por fases (ver figura 1) que se concretan en siete sesiones, cuyas actividades asumen un carácter regulador, propiciando un clima de aula adecuado que facilita las interacciones afectivas.

\section{Figura 1}

Fases de la intervención indagatoria y metaemocional

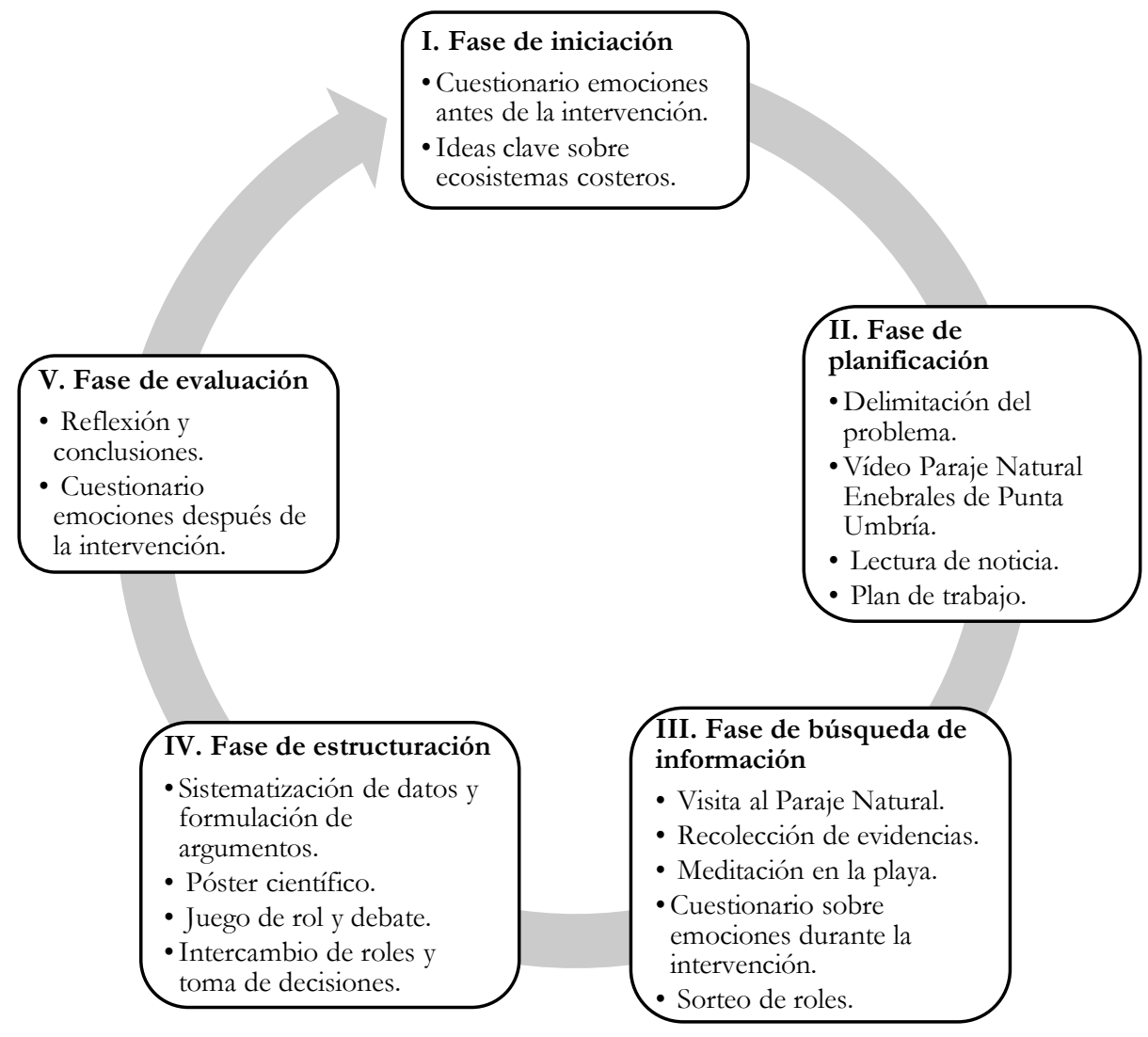

Desde una perspectiva sociológica, este problema supone que las personas parten de premisas diversas, involucran un número importante de individuos o grupos cuyas diferencias son abiertas y visibles. Prueba 
de ello, el debate generado entre diversos sectores de la comunidad, aquellos que vislumbraban el proyecto turístico como oportunidad para potenciar la economía, y por otra parte, los detractores a esta idea, quienes defendían las necesidades de la protección ambiental. De esta manera, el problema es controvertido pues no puede resolverse definitivamente a partir de la prueba científica, ya que también se puede llegar a un consenso social apelando a un punto de vista que generalmente se considera como sentido común (Crick, 1998). Precisamente, las manifestaciones pueden derivarse de emociones, pues como señala Claxton (1991) las personas pueden ser resistentes al cambio incluso cuando la evidencia es convincente. Entonces, se comienza con un acercamiento al contexto a través de la visita al ecosistema y el análisis de noticias específicas que permiten identificar los aspectos controvertidos y las ideas científicas clave. Más adelante, se promueve la discusión con miras a la participación en un juego de rol, estrategia didáctica exigente para los estudiantes, pues deben exponer interés y comprensión del por qué el tema se considera relevante, distinguir información científica clave, superar creencias ingenuas y utilizar apropiadamente el razonamiento científico. En este escenario, la formadora organiza la discusión para regular las interacciones sociales, pues influyen en los esquemas de argumentación grupal. De esta manera, evita conflictos entre los estudiantes y promueve la reflexión sobre sus puntos de vista, escuchando lo que cada uno tiene que decir. Además, siguiendo a Garritz (2010), la formadora motiva a los estudiantes para que identifiquen los involucrados en el problema, sus intereses, las intenciones que guían las decisiones, beneficiados o marginados, entre otros aspectos.

\subsection{Instrumentos, procedimientos de recolección y análisis de datos}

Se elaboró un cuestionario con escala Likert de cinco niveles para recoger el grado de acuerdo en la intensidad de siete emociones positivas (entusiasmo, satisfacción, interés, aceptación, tranquilidad, bienestar y asombro) y siete emociones negativas (susto, temor, enojo, tensión, frustración, aburrimiento y rechazo) que experimentan los futuros maestros hacia la formadora en el transcurso de la intervención (ver anexo I). La administración se realizó en octubre, diciembre y enero del curso 2016-2017, asegurando confidencialidad y anonimato. La fiabilidad de la escala representada en el alfa de Cronbach resultó satisfactoria en los tres momentos tanto para las emociones negativas $(, 90,74,, 83)$ como para las emociones positivas $(, 81,78,, 76)$ respectivamente, en coherencia con otros estudios que emplean cinco niveles asociados a fiabilidad alta (Matas, 2018). El análisis factorial confirmatorio aplicado para un solo factor, registró una varianza explicada del 63,0\%, con un valor ,906 para el KMO y ,000 para la esfericidad de Barlett, cifras sobresalientes en Ciencias Sociales. El omega de McDonald suministró un valor de ,94, también muy robusto y superior al alfa de Cronbach. Por otra parte, dada la carencia de normalidad $(\mathrm{p}=, 00)$, se realizó la prueba H de Kruskal Wallis para tres momentos independientes al 95,0\% de confianza $(\mathrm{p}<, 05)$, con el fin de determinar diferencias estadísticas. También, se calculó el error tipo II ( $\beta$ ), la potencia y el tamaño del efecto para estimar la magnitud de tales contrastes.

En términos cualitativos, se elaboró un sistema de categorías para el análisis de la reflexión declarativa de la formadora (ver tabla 2 más adelante), obtenida a través de una entrevista semiestructurada. En particular, es de nuestro interés enfatizar en las concepciones y emociones de la formadora, dado que en el marco del modelo propuesto por Gess-Newsome (2015) amplifican o filtran los conocimientos profesionales asociados a los tópicos científicos que se llevarán a la planificación y práctica. Cada subcategoría presenta al menos tres ideas analíticas con un grado de complejidad creciente en la propia reflexión a las que hace referencia a la dimensión técnica, práctica y crítica de la Hipótesis de la Complejidad. La complejidad se concibe como la evolución de la capacidad del profesor de interactuar con el entorno educativo, social y natural, en el ejercicio de su profesión y a través de la reflexión orientada hacia y en la práctica, afectando los aspectos ideológicos, educativos, contextuales, epistemológicos, curriculares y emocionales de su trabajo y pensamiento (Vázquez-Bernal et al., 2007, 2010, 2012).

En el análisis de contenido, llevado a cabo con el programa AQUAD 7, se realizó la codificación por líneas en el texto identificando las unidades que distinguen algún descriptor en una dimensión particular y relacionándolo con un código apropiado. Seguidamente, se intentó la reconstrucción del sistema de significados de la formadora, para lo cual se extrajeron referencias que sirven como evidencia en las declaraciones presentes en el texto. Por último, se realiza la triangulación a partir de la aplicación de otros instrumentos de primer y segundo orden (tabla 1), tales como la observación participante del investigador, las narrativas de los estudiantes, las percepciones de la profesora novel y la literatura. 
Tabla 1

Instrumentos de recogida de datos, análisis y presentación de datos

\begin{tabular}{lllll}
\hline $\begin{array}{l}\text { Instrumentos de } \\
\text { Primer Orden }\end{array}$ & $\begin{array}{l}\text { Instrumentos } \\
\text { de Segundo } \\
\text { Orden }\end{array}$ & Participantes & $\begin{array}{l}\text { Foco de la } \\
\text { indagación }\end{array}$ & $\begin{array}{l}\text { Técnicas de } \\
\text { análisis }\end{array}$ \\
\hline $\begin{array}{l}\text { Guión de entrevista } \\
\text { semiestructurada a } \\
\text { formadora }\end{array}$ & $\begin{array}{l}\text { Sistema de } \\
\text { categorías }\end{array}$ & Formadora & $\begin{array}{l}\text { Interacción } \\
\text { emocional }\end{array}$ & $\begin{array}{l}\text { Análisis de } \\
\text { frecuencias y } \\
\text { contenido }\end{array}$ \\
$\begin{array}{l}\text { Diario de } \\
\text { observación del }\end{array}$ & & & & \\
$\begin{array}{l}\text { Guión de entrevistador } \\
\text { a profesora novel }\end{array}$ & & Profesora novel & Percepción & \\
Narrativas & Fucia la \\
& formadora & \\
\hline
\end{tabular}

Tabla 2

Sistema de categorías para el análisis de la reflexión de la formadora en el ámbito amplificadores y filtros

\begin{tabular}{|c|c|c|c|c|}
\hline Categoría & Subcategorías & Descriptores* & Códigos & Referencias \\
\hline Concepciones & $\begin{array}{c}\text { Concepciones } \\
\text { sobre la } \\
\text { indagación }\end{array}$ & $\begin{array}{c}\text { DT: En cuanto a la implementación } \\
\text { de la indagación, el profesorado en } \\
\text { ejercicio encuentra obstáculos como } \\
\text { ausencia de materiales o falta de } \\
\text { tiempo para desarrollar el plan de } \\
\text { estudios. } \\
\text { DT: El profesorado relaciona el } \\
\text { aprendizaje por investigación con } \\
\text { actividades divertidas para los } \\
\text { estudiantes, es decir, tiene un } \\
\text { carácter más motivador que } \\
\text { potenciador del aprendizaje. } \\
\text { DP: El profesorado considera } \\
\text { necesaria la actualización de sus } \\
\text { metodologías docentes o los } \\
\text { conocimientos sobre la materia a } \\
\text { impartir y trabajar desde el } \\
\text { planteamiento de problemas para } \\
\text { desarrollar la competencia de } \\
\text { aprender a aprender. El alumno } \\
\text { asume un rol protagonista. } \\
\text { DC: El profesorado prepara } \\
\text { materiales, incluye actividades de } \\
\text { indagación, reflexiona sobre su } \\
\text { implementación y transferencia a } \\
\text { contextos que trascienden al aula. }\end{array}$ & $\begin{array}{l}\text { PACT, } \\
\text { PAPA, } \\
\text { PPTG }\end{array}$ & $\begin{array}{c}\text { Abril et al. } \\
\text { (2013) }\end{array}$ \\
\hline Emociones & $\begin{array}{l}\text { Emociones en } \\
\text { la enseñanza }\end{array}$ & $\begin{array}{l}\text { DT: Las emociones negativas del } \\
\text { profesor se relacionan con el mal } \\
\text { comportamiento de los estudiantes o } \\
\text { la ausencia de disciplina en el aula, } \\
\text { incrementando el riesgo del burnout. } \\
\text { DP: Las emociones positivas del } \\
\text { profesor son esenciales para su } \\
\text { propio bienestar y también pueden } \\
\text { incidir en el bienestar de los }\end{array}$ & PPOS & $\begin{array}{l}\text { Hagenauer et } \\
\text { al. (2015), } \\
\text { Hargreaves } \\
\text { (1998), } \\
\text { Shapiro } \\
\text { (2010), } \\
\text { Sutton y } \\
\text { Harper }\end{array}$ \\
\hline
\end{tabular}




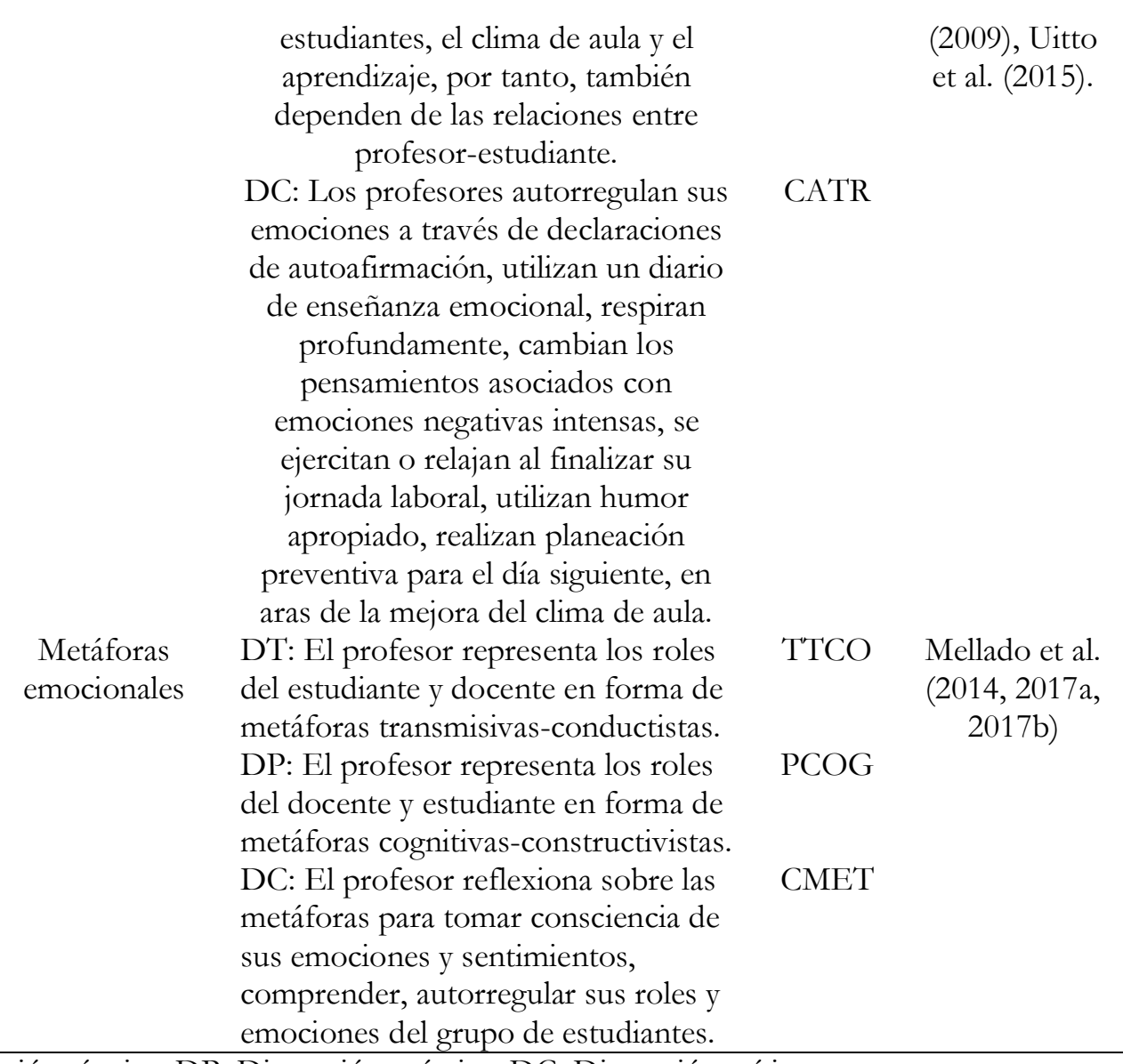

Nota: *DT: Dimensión técnica, DP: Dimensión práctica, DC: Dimensión crítica.

\section{Resultados y discusión}

\subsection{Análisis descriptivo e inferencial del cambio en las emociones}

Como se aprecia en la figura 2 y tabla 3 , los resultados indican el predominio de las emociones positivas respecto a la formadora en el transcurso de la intervención. En trabajos previos (Retana-Alvarado et al., 2017, 2018, 2019a, 2019b) mostramos resultados similares en este sentido, aunque centrados en la asignatura, los contenidos científicos y el clima de aula.

$\mathrm{Al}$ comienzo, todas las emociones negativas fueron más intensas en contraste con los siguientes momentos, especialmente tensión y temor. Más adelante, todas las emociones negativas disminuyeron, en particular, temor, frustración, aburrimiento y rechazo con tamaños de efecto pequeños. En ese momento, tensión y aburrimiento alcanzaron los promedios más altos. Finalmente, todas las emociones negativas menos aburrimiento experimentaron un incremento, en particular susto, temor y tensión, pero esos cambios no son significativos. Al comparar el momento previo y final se evidencia que todas las emociones negativas disminuyeron, siendo aburrimiento y rechazo las que experimentaron la caída más considerable, cuyos tamaños de efecto son pequeños. De nuevo, tensión registra la intensidad más alta en cada momento; por tanto, es un filtro de los estudiantes que actúa como factor limitante en su interacción con la formadora.

En cuanto a las emociones positivas, aceptación, interés y tranquilidad fueron las más intensas antes de la intervención. Más adelante, todas registraron mayor intensidad en el intermedio en comparación con el inicial. Así, entusiasmo, satisfacción, interés, tranquilidad, bienestar y asombro manifestaron el incremento más expresivo (tamaños de efecto medianos y altos). En ese instante, interés y tranquilidad alcanzaron las intensidades más altas.

Finalmente, mermó la mayoría de las emociones positivas, especialmente tranquilidad; por su parte, satisfacción, aceptación y bienestar expresaron un pequeño incremento a favor del momento final, ubicándose satisfacción, interés y aceptación como las emociones más intensas. Por último, entre el antes y después, todas las emociones positivas incrementaron, aunque de manera significativa entusiasmo, satisfacción, interés, tranquilidad, bienestar y asombro con tamaños de efecto medianos y altos. 
Figura 2

Cambio de las emociones hacia la formadora

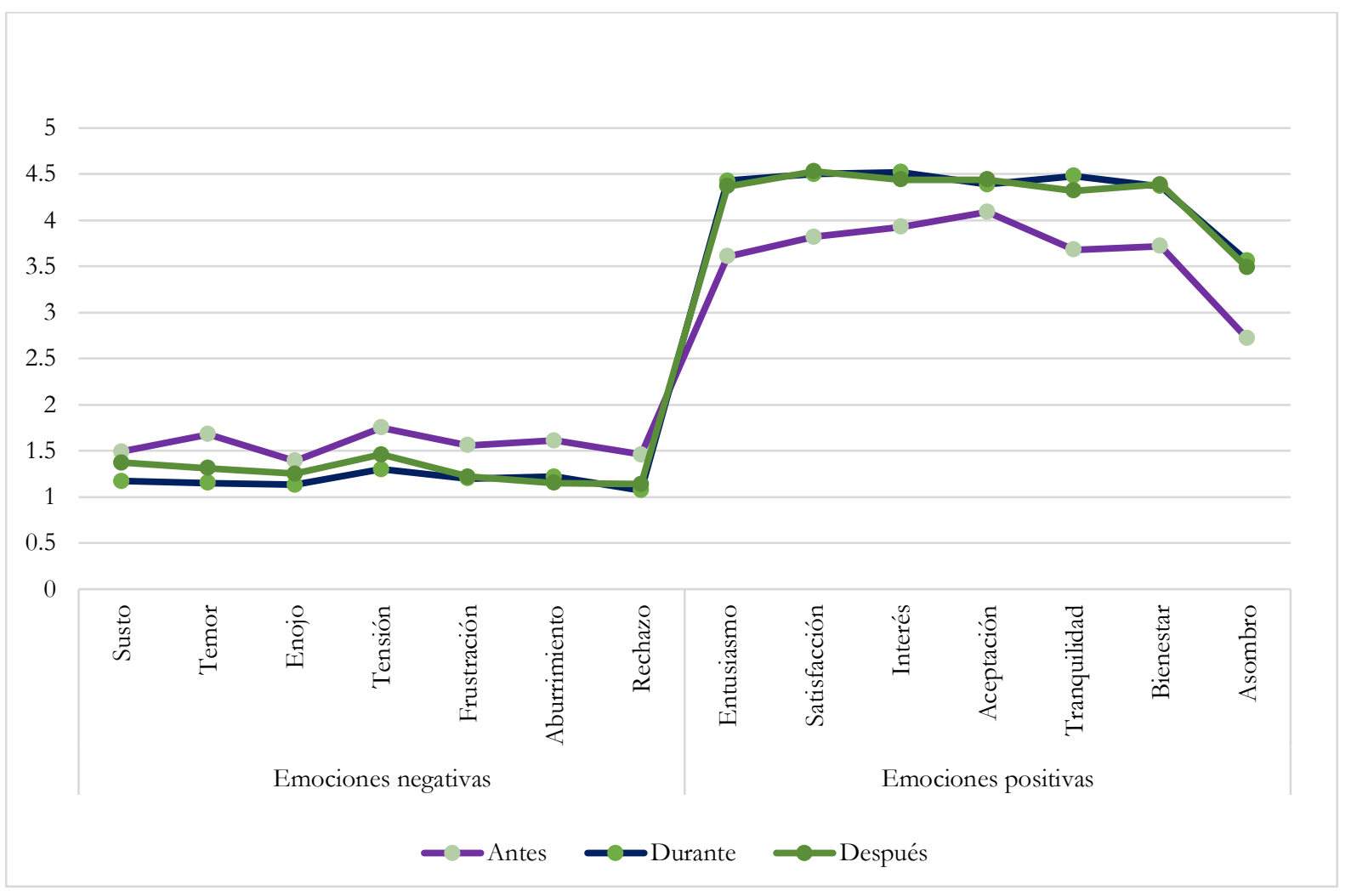

Tabla 3

Prueba no paramétrica $H$ de Kruskal Wallis para las emociones positivas y negativas hacia la formadora en función de los momentos de la intervención

\begin{tabular}{|c|c|c|c|c|c|c|c|}
\hline Variable & $\begin{array}{c}\text { Muestra 1-Muestra } \\
2\end{array}$ & $X^{2}$ & $U$ & $p$ & $\beta$ & $P$ & $T E$ \\
\hline \multirow[t]{3}{*}{ Susto } & Antes-Durante & - & 1220,0 & - & 21,8 & 78,2 & ,42 \\
\hline & Antes-Después & - & 1351,0 & - & 80,2 & 19,8 & ,46 \\
\hline & Durante-Después & - & 1585,0 & - & 49,8 & 50,2 &, 54 \\
\hline \multirow[t]{3}{*}{ Temor } & Antes-Durante & 19,61 & 1105,5 &, $008 *$ & 5,50 & 94,5 & ,38 \\
\hline & Antes-Después & 14,97 & 1188,0 &, 067 & 28,2 & 71,8 & ,41 \\
\hline & Durante-Después & $-4,64$ & 1542,0 & 1,000 & 69,6 & 30,4 &, 53 \\
\hline \multirow[t]{3}{*}{ Enojo } & Antes-Durante & - & 1245,5 & - & 39,5 & 60,5 & ,43 \\
\hline & Antes-Después & - & 1372,5 & - & 72,5 & 27,5 & 47 \\
\hline & Durante-Después & - & 1587,5 & - & 71,6 & 28,4 &, 54 \\
\hline \multirow[t]{3}{*}{ Tensión } & Antes-Durante & 19,3 & 1113,0 &, 021 & 13,9 & 86,1 & ,38 \\
\hline & Antes-Después & 13,71 & 1208,5 &, 167 & 42,6 & 57,4 & ,41 \\
\hline & Durante-Después & $-5,60$ & 1561,5 & 1,000 & 78,6 & 21,4 &, 54 \\
\hline \multirow[t]{3}{*}{ Frustración } & Antes-Durante & 17,0 & 1151,0 & ,030* & 19,3 & 80,7 & ,39 \\
\hline & Antes-Después & 18,3 & 1129,0 &, 017 & 21,4 & 78,6 & 39 \\
\hline & Durante-Después & 1,33 & 1433,3 & 1,000 & 94,9 & 5,10 & ,49 \\
\hline \multirow[t]{3}{*}{ Aburrimiento } & Antes-Durante & 21,1 & 1079,0 & ,006* & 7,20 & 92,8 & 37 \\
\hline & Antes-Después & 25,9 & 991,0 & ,000* &, 70 & 99,3 &, 34 \\
\hline & Durante-Después & 4,89 & 1370,0 & 1,000 & 71,0 & 29,0 & 47 \\
\hline \multirow[t]{3}{*}{ Rechazo } & Antes-Durante & 18,8 & 18,75 &, $003 *$ & 4,60 & 95,4 &, 003 \\
\hline & Antes-Después & 15,6 & 1177,5 &, $020^{*}$ & 22,9 & 77,1 &, 40 \\
\hline & Durante-Después & $-3,14$ & 1514,0 & 1,000 & 72,3 & 27,7 &, 52 \\
\hline \multirow[t]{3}{*}{ Entusiasmo } & Antes-Durante & $-39,8$ & 2176,5 &, $000 *$ & 0,00 & 100 &, 75 \\
\hline & Antes-Después & $-37,4$ & 2129,0 &, $000 *$ & 20 & 99,8 &, 73 \\
\hline & Durante-Después & 2,36 & 1418,0 & 1,000 & 88,5 & 11,5 & ,49 \\
\hline
\end{tabular}




\begin{tabular}{cccccccc} 
Satisfacción & Antes-Durante & $-35,8$ & 2103,0 &, $000^{*}$ &, 10 & 99,9 &, 72 \\
& Antes-Después & $-37,9$ & 2140,0 &, $000^{*}$ & 0,00 & 100 &, 73 \\
& Durante-Después & $-2,07$ & 1495,5 & 1,000 & 90,2 & 9,80 &, 51 \\
Interés & Antes-Durante & $-32,4$ & 2031,0 &, $000^{*}$ &, 40 & 99,6 &, 70 \\
& Antes-Después & $-25,8$ & 1933,5 &, $005^{*}$ & 2,60 & 97,4 &, 66 \\
& Durante-Después & 6,62 & 1328,0 & 1,000 & 79,6 & 20,4 &, 46 \\
\multirow{5}{*}{ Aceptación } & Antes-Durante & $-16,8$ & 1767,5 &, 125 & 24,8 & 75,2 &, 61 \\
& Antes-Después & $-19,0$ & 1795,0 &, 064 & 23,1 & 76,9 &, 62 \\
& Durante-Después & $-2,21$ & 1504,0 & 1,000 & 93,1 & 6,90 &, 52 \\
\multirow{5}{*}{ Biennestar } & Antes-Durante & $-35,8$ & 2090,0 &, $000^{*}$ &, 10 & 99,9 &, 72 \\
& Antes-Después & $-27,6$ & 1968,0 &, $003^{*}$ & 2,10 & 97,9 &, 67 \\
& Durante-Después & 8,22 & 1297,0 &, 985 & 70,1 & 29,9 &, 44 \\
& Antes-Durante & 21,1 & 1996,5 &, $006^{*}$ & 1,60 & 98,4 &, 68 \\
& Antes-Después & 25,9 & 2016,0 &, $000^{*}$ & 1,10 & 98,9 &, 69 \\
& Durante-Después & 4,88 & 1471,5 & 1,000 & 93,3 & 6,70 &, 50 \\
& Antes-Durante & $-28,3$ & 1969,0 &, $004 *$ & 6,20 & 93,8 &, 68 \\
& Antes-Después & $-24,4$ & 1896,5 &, $016^{*}$ & 15,2 & 84,8 &, 65 \\
& Durante-Después & 3,88 & 3,880 & 1,000 & 88,4 & 11,6 &, 001 \\
\hline
\end{tabular}

Nota: $* p<, 05$

\subsection{Análisis de la reflexión de la formadora}

\section{Análisis de frecuencias}

A partir del análisis de la entrevista a la formadora, en la tabla 4 se exponen, entre paréntesis, las frecuencias de las codificaciones encontradas correspondientes a las subcategorías, en correspondencia con las dimensiones de la Hipótesis de la Complejidad. Los hallazgos sugieren un mayor predominio de códigos prácticos (6) y críticos (7) en la reflexión, asociados mayoritariamente a las concepciones y metáforas emocionales.

Tabla 4

Distribución de frecuencias de codificaciones en cada dimensión

\begin{tabular}{cccc}
\hline Subcategorías & Dimensión Técnica & Dimensión Práctica & Dimensión Crítica \\
\hline Concepciones & TAMT(0), TMOT $(0)$ & PACT (0), PAPA (3), PPTG & CREG (3) \\
& & $(1)$ & \\
Emociones & TNEG $(0)$ & PPOS $(2)$ & CATR $(0)$ \\
Metáforas & TTCO $(0)$ & PCOG $(0)$ & \\
emocionales & & & \\
\hline
\end{tabular}

\section{Análisis de la teoría implícita}

Seguidamente, se exploran los significados o contenido latente considerando la estructura del modelo dinámico de conocimiento profesional y habilidad, mostrando segmentos de texto codificados para cada dimensión.

Ámbito: amplificadores y filtros de la profesora.

Categoría: concepciones.

Dimensión técnica: no se encuentran unidades de análisis referentes a obstáculos como carencia de materiales y tiempo para realizar indagación (TAMT), tampoco sobre la concepción didáctica del profesorado que relaciona el aprendizaje por investigación como una actividad divertida con carácter motivador (TMOT).

Dimensión práctica: se hallan tres citas en la subcategoría concepciones sobre indagación que resaltan el trabajo a partir de problemas para alcanzar la competencia aprender a aprender (PAPA), por ejemplo:

(558-561: PAPA) Conseguimos que nuestros alumnos tengan por objetivo aprender, es decir, se generen más cuestiones de las que se han generado en la propia aula»

En esta dimensión, también toma valor el rol protagonista que se le atribuye al estudiante (PPTG): 
(806-808: PPTG) Es mucho más formativo para el alumno el tema de hacerlo protagonista.

Dimensión crítica: Si bien, en las observaciones del investigador, las narrativas de los estudiantes y la entrevista a la profesora novel se aprecia una planificación y acción indagatoria en la práctica, en el caso de la entrevista a la formadora experta no se desprenden referencias directas que destaquen la inclusión de actividades indagatorias en la programación didáctica, así como su puesta a punto y transferencia a otros contextos (CREF). Al respecto, la implementación de la indagación salta a la luz en la triangulación.

Categoría: emociones.

Dimensión técnica: no existen referencias en relación con la manifestación de emociones negativas por parte de la profesora (TNEG) ni la representación de los roles de estudiantes y docente en forma de metáforas emocionales transmisivas (TTCO).

Dimensión práctica: se encuentran dos unidades de análisis que informan acerca de las emociones positivas que experimenta la profesora (PPOS), tales como bienestar, disfrute y tranquilidad; afectos que la formadora transmite en la clase de manera implícita o a través de la sonrisa.

(893-898: PPOS) Sentirme bien cuando estoy con ellos, intentar sonreír, todo este tipo de cuestiones que tú no se las estás transmitiendo de forma explícita, pero, están captando de alguna manera.

(1014-1018: PPOS) Mi sensación es muy buena, es de tranquilidad, de ir a clase a disfrutar y la sensación muy buena, lo cual no quita que yo tenga que mejorar.

Dimensión crítica: no existen referencias asociadas con emociones en la enseñanza para esta dimensión. Aunque sí hay cuatro unidades de análisis que refieren a las metáforas emocionales. En este sentido, la formadora reflexiona sobre las metáforas para concienciarse de sus emociones y autorregularlas en la clase (CMET). Por ejemplo, desde la metáfora «ojos de abeja, ojos de mosca» asume una actitud positiva hacia los estudiantes y considera que todos tienen algo favorable que pueden aportar.

(953-963: CMET) Pues, la metáfora "ojos de abeja ojos de mosca", yo en clase intento tener ojos de abeja. Es decir, ver lo positivo porque de los setenta alumnos que tengo allí seguro que de todos algo positivo puedo sacar. Entonces, ver que todos tienen algo que aportar, intentar no aislar a nadie, sino todo lo contrario.

Así que es un facilitador del cambio para alcanzar competencias emocionales:

(976-978: CMET) Ese es el cambio que tenemos que dar para llegar a ser emocionalmente competentes.

Refiriéndose a los estudiantes, la profesora menciona lo siguiente: (985-989: CMET) Ellos también han sabido sacar lo positivo, los comparo con lo mismo, pues yo no he tenido ninguna sensación de que se hayan sentido mal sino todo lo contario.

\subsection{Triangulación de resultados}

De todo lo anterior, se desprende una mejora en las emociones positivas y una disminución considerable de las emociones negativas.

\section{Perfil afectivo de la formadora e interacción emocional}

En primer lugar, como se infiere de la entrevista, posiblemente se debe a la madurez emocional de la formadora, quien es consciente de sus emociones, transfiriéndolas desde su propio modelo didáctico a los futuros maestros para la conformación de un clima de aula favorable que afirma seguridad, tranquilidad y bienestar en las interacciones. De acuerdo con Newberry (2010), las relaciones entre profesor y estudiantes determinan un clima de aula, donde el rango de necesidades y emociones académicas es un desafío para el profesorado. La autora propone que la reflexión y discusión guiada facilita el proceso de negociación para mantener relaciones positivas. En virtud de ello, la actividad emocional es gestionada a través del respeto hacia la integridad y las autoafirmaciones positivas como se ejemplifica en las siguientes citas:

(Entrevista a formadora: 885-898: CAFÉ, CHUM, CPOS, PPOS) Es muy importante cómo tú seas emocionalmente para trabajar con las personas. Entonces, tratar a las personas que tengo allí como personas, intentar trabajar siempre desde lo positivo, transmitirles seguridad, tranquilidad y buen clima.

(Registro de observación del investigador: 28 de setiembre de 2016) La profesora comenta que las relaciones sociales son muy importantes para el bienestar. Esos hábitos saludables deben transmitirse desde el modelo del profesor.

(Narrativa de estudiante: Hugo) Esto ha sido posible gracias al clima de diálogo y cercanía que se ha establecido en clase, cosa que, sobre todo en la universidad, es raro y al mismo tiempo se agradece. 
Entonces se confirma una interacción positiva debido a las características particulares de la formadora y de los estudiantes que facilita la participación y el aprendizaje. Según Brinkworth et al. (2018) las relaciones entre profesores y estudiantes se encuentran en el corazón del entorno de aprendizaje. Dado que las aulas son fundamentalmente contextos sociales, sostienen que las intervenciones enfocadas en las interacciones son prometedoras para optimizar los resultados de los estudiantes, dado que aportan una comprensión más precisa de la naturaleza de las relaciones, cómo cambian en el tiempo y cómo mejoran.

(Entrevista a profesora novel, 31 de mayo de 2017) -Sobre la profesora todo positivo. Pues me encantó la forma de las clases, me resultó muy novedoso, me gustó la relación alumno-profesora. Los alumnos, también positivos. Pero, era un grupo muy bueno que participaba mucho, muy activo, con mucho que aprender.

Desde luego, la percepción es favorable respecto a las emociones que exhiben los estudiantes en el proceso formativo:

(Entrevista a formadora: 985-986, CMET/987-989/1000) -Ellos también han sabido sacar lo positivo. Yo no he tenido ninguna sensación de que se hayan sentido mal, sino todo lo contario.

\section{Labor emocional}

En segundo lugar, como se deriva de las declaraciones de los estudiantes, al parecer la formadora posee vocación para la enseñanza de las ciencias que se traduce en labor emocional, pues manifiesta interés para apoyar a los estudiantes ante cualquier dificultad. De acuerdo con Fajet et al. (2005), los maestros en formación inicial conciben la enseñanza principalmente como una tarea que involucra relaciones afectivas e interpersonales más que como una profesión que requiere un profesional capacitado y con conocimientos. También, como se deduce de la entrevista, la profesora reflexiona sobre la progresión en el aprendizaje profesional, facilita la participación, el trabajo colaborativo, la discusión y el humor.

(Narrativa de estudiante: Hugo) Uno de los puntos de mayor relevancia que destacaría en esta reflexión sería la labor de nuestra docente, lo digo porque creo firmemente que encontrar un docente con esa vocación es una verdadera tarea de investigación. Con vocación me quiero referir a esas ganas de ver y observar cómo sus alumnos aprenden de verdad, participan en clase, trabajan en equipo, debaten, ríen, se divierten, se enfadan... Es realmente agradable ver cómo una profesora se interesa por sus alumnos, cómo ante cualquier dificultad o adversidad por pequeña que sea, está ahí para resolverla. Estas pequeñas cosas, que parecen insignificantes, son las que realmente diferencian a unos profesores de otros, las que hacen que los alumnos valoremos el trabajo y el esfuerzo realizado, mostremos verdadero interés y respeto tanto por las clases como por la labor del docente.

\section{Indagación}

En tercer lugar, la formadora es percibida por los futuros maestros como una facilitadora que acerca los contenidos científicos a las experiencias de la cotidianidad mediante una metodología que les provoca motivación e interés, incluso ganas de asistir a clases. La comprensión de los tópicos se amplifica debido a la reflexión sobre las actividades indagatorias que se realizaban en la clase.

(Narrativa de estudiante: Lucas) En lo que respecta a la profesora, ha sido una persona que nos ha puesto muchas facilidades y siempre se ha interesado por que nos quedase claro todo lo que explicaba. Además, ha hecho que las clases fuesen motivantes y lúdicas, siempre ha intentado acercarnos la materia a nuestras experiencias más cercanas de la vida cotidiana, para que la entendiésemos con más facilidad.

(Narrativa de estudiante: Elif) Debo reconocer que en cuanto supe que tenía que cursar esta asignatura, pensé de forma negativa pues es una de las que no me gustaba durante mi etapa en Educación Primaria. En los primeros días de la asignatura salía del aula pensando de igual forma, inclusive hubo un día que lo pasé fatal. Pienso que los motivos de que mis pensamientos fuesen negativos radican en la forma en la cual me impartieron estos contenidos en primaria: todo muy tradicional, basado en la memorización y sin tener en cuenta los intereses del alumnado. A medida que fueron pasando las clases, yo misma noté un cambio de actitud hacia la asignatura pues asistía con ganas, motivación e interés por aprender. Este cambio lo atribuyo a la profesora (de forma positiva) y su manera de impartir las clases, pues se interesó en que experimentáramos, descubriéramos y reflexionáramos sobre las actividades que hacíamos, que aprendiéramos de nuestra experiencia.

Significa entonces que las causas que generan el cambio, posiblemente se corresponden con el perfil vocacional y afectivo de la formadora, quien promueve relaciones auténticas con los futuros maestros a 
través de su labor emocional, transfiriendo emociones positivas en el clima de aula. También, facilita los contenidos científicos con las experiencias cotidianas y la reflexión sobre las actividades indagatorias contribuyendo en un incremento de la motivación y el interés.

\section{Conclusiones e implicaciones}

El propósito de este artículo ha sido analizar el cambio en la intensidad de las emociones de maestros en formación inicial hacia una profesora, en el transcurso de una asignatura de Didáctica de las Ciencias con una intervención indagatoria sobre una cuestión sociocientífica controvertida. En este sentido, hemos analizado las emociones que se exhiben al comienzo, durante y después del curso; los cambios detectados; así como el establecimiento de las causas.

Considerando las preguntas de investigación formuladas:

¿Qué emociones positivas y negativas se manifiestan hacia la formadora? ¿Qué variaciones se detectan en función del momento de la intervención?

Los resultados permiten concluir que entre el antes y durante hay un incremento significativo para entusiasmo, satisfacción, interés, tranquilidad, bienestar y asombro. Entre el durante y después se evidencia un incremento en satisfacción, aceptación y bienestar, así como una disminución en entusiasmo, interés, tranquilidad y asombro. Entre el antes y después se detecta un incremento significativo para entusiasmo, satisfacción, interés, tranquilidad, bienestar y asombro.

Por otra parte, entre el antes y durante disminuyeron todas las emociones negativas; dicho cambio es significativo para temor, frustración, aburrimiento y rechazo. Entre el durante y después hay un incremento en susto, temor, enojo, tensión, frustración y rechazo; asimismo, se reporta un descenso en el aburrimiento. Entre el antes y después, se detecta una caída en todas las emociones, siendo significativa para aburrimiento y rechazo. Estos hallazgos son meridianamente coincidentes con otros estudios que registran un incremento de las emociones positivas y un descenso en las emociones negativas en intervenciones alternativas (Jeong et al., 2016; Marcos-Merino et al., 2020).

¿Cuáles son las posibles causas que facilitan el cambio?

Como se infiere de las declaraciones de los estudiantes, de la profesora novel y la observación del investigador, el cambio emocional en referencia a la profesora puede que esté causado por sus características personales y profesionales que inspiran tranquilidad, paciencia, disponibilidad y humor, en un clima de participación donde todos pueden aportar sin miedo a reacciones coercitivas, puesto que concibe el error como una forma de aprender y reflexionar. Entonces, los futuros maestros aprovechan estos facilitadores para mantener ese ambiente interactivo.

Además, como se deduce de la entrevista, la formadora no se limita a impartir contenidos a través de una metodología indagatoria, sino que desarrolla una labor emocional consciente basada en el fortalecimiento de las emociones positivas a partir del empleo de estrategias autorregulatorias, que a su vez, favorecen la transferencia y las interacciones. Según Sutton y Harper (2009), la huella de un maestro eficaz está construida en un ambiente de aprendizaje productivo utilizando el humor y la expresión de emociones positivas. Estos hallazgos son coincidentes con el estudio de Hagenauer y Volet (2014), cuyos resultados indican que los docentes universitarios disfrutan de las clases en las que las relaciones entre profesor y estudiantes se perciben como productivas.

Por último, como se infiere de la entrevista, la formadora posee una actitud positiva hacia los estudiantes y sus necesidades, ha desarrollado habilidades de regulación emocional mediante procesos de autoformación, diálogos con colegas y reflexión sobre la práctica. Creemos que estas características podrían fundamentar la construcción de un modelo de profesor que sirva de referencia para la formación docente (Jiménez-Pérez y Wamba, 2004).

Por otra parte, como señalamos en otros trabajos (Retana-Alvarado et al., 2018, 2019a, 2019b), una de las implicaciones didácticas más relevantes que se derivan de esta investigación consiste en el abordaje integrado de la educación emocional con las ideas científicas clave, en el marco de un modelo de conocimiento profesional promotor de la evolución del CDC para la futura enseñanza. Según Badillo et al. (2016), las sinergias entre estrategias formativas como el uso de materiales ejemplares, observación de clasesmodelo, análisis didáctico de vídeo-episodios de aula y discusión conjunta tienen mayor potencial para incidir en el CDC de los maestros. Bajo la perspectiva de la Hipótesis de la Complejidad, sostenemos que en el contexto natural de formación docente, el formador debe representar un modelo personal y profesional de referencia que sea replicable y transferible a las aulas de Educación Primaria. Por tanto, se espera que el futuro maestro asuma un perfil afectivo robusto junto con concepciones y prácticas coherentes con la dimensión crítica, donde los problemas de la práctica asuman un carácter transformador y trasciendan el 
contexto escolar para la mejora de las competencias emocionales que contribuyan en la formación integral de la persona (física, cognitiva, emocional, social, moral y espiritual), así como la creación de climas emocionales que favorezcan la felicidad y le capaciten para la vida (Bisquerra y Pérez-Escoda, 2007).

Finalmente, siguiendo a Vázquez-Bernal et al. (2012) se hace más patente la necesidad de incursionar en estudios de caso con formadores en Didáctica de las Ciencias Experimentales, con el propósito de profundizar en aquellas interacciones emocionales capaces de paliar las emociones negativas y superar aquellos obstáculos ideológicos, afectivos, cognitivos y curriculares que dificultan el desarrollo profesional. Eso conduciría a otros estudios sobre la dimensión afectiva con niñas y niños de Primaria. Estas perspectivas abren nuevos puentes para fortalecer las relaciones académicas internacionales y representan un faro que ilumina el cambio educativo, especialmente en contextos que demandan innovación didáctica para asegurar el bienestar personal y social.

\section{Apoyos}

El estudio ha sido financiado por la Universidad de Costa Rica. El primer autor agradece el aporte académico a la Universidad de Huelva, España.

\section{Referencias}

Abril, A. M., Romero, M. Quesada, A. y García, F. J. (2013). Creencias del profesorado en ejercicio y en formación sobre el aprendizaje por investigación. Revista Eureka sobre Enseñanza y Divulgación de las Ciencias, 11(1), 22-33.

Badillo, E., Moreno, I. y Couso, D. (2016). ¿Cómo incidir en el conocimiento didáctico del contenido?: Análisis de una intervención formativa en maestros de matemática en ejercicio. En G. A. Perafán, E. Badillo y A. Adúriz-Bravo (Eds.), Conocimiento y emociones del profesorado. Contribuciones para su desarrollo e implicaciones didácticas (pp. 155-182). Editorial Aula de Humanidades.

Bell, B. y Gilbert, J. (1994). Teacher development as professional, personal, and social development. Teacbing and Teacher Education, 10(5), 483-497. DOI: https: 10.1016/0742-051X(94)90002-7

Bisquerra, R., y Pérez-Escoda, N. (2007). Las competencias emocionales. Educación XXI, 10, 61-82.

Brinkworth, M. E., McIntyre, J., Juraschek, A. D. y Gehlbach, H. (2018). Teacher-student relationships: The positives and negatives of assessing both perspectives. Journal of Applied Developmental Psychology, 55, 2438. DOI: $10.1016 /$ j.appdev.2017.09.002

Cañal, P., Pozuelos, F. J. y Travé, G. (2005). Proyecto curricular Investigando Nuestro Mundo. Descripción general y fundamentos. Diada.

Carlson, J. y Daehler, K. R. (2019). The Refined Consensus Model of Pedagogical Content Knowledge in Science Education. En A. Hume, R. Cooper, A. Borowski (Eds.), Repositioning Pedagogical Content Knowledge in Teachers Knowledge for Teaching Science (pp. 77-94). Singapore.

Chang, M. L. y Davis, H. A. (2009). Understanding the role of teacher appraisals in shaping the dynamics of their relationships with students: deconstructing teachers' judgments of disruptive behavior/students. En P. A. Schutz y M. Zembylas (Eds.), Advances in teacher emotion research (pp. 95-127). Springer.

Claxton, G. (1991). Educating the enquiring mind: The challenge for school science. Harvester Wheatsheaf.

Crick, B. (1998). Education for citizenship and the teaching of democracy in schools. Qualifications and Curriculum Authority.

Fajet, W., Bello, M., Leftwich, S. A., Mesler, J. L. y Shaver, A. N. (2005). Pre-service teachers' perceptions in beginning education classes. Teaching and Teacher Education, 21(6), 717-727. DOI: 10.1016/j.tate.2005.05.002

Frenzel, A. C., Pekrun, R., Goetz, T., Daniels, L. M. y Durksen, T. L. (2016). Measuring Teachers’ enjoyment, anger, and anxiety: The Teacher Emotion Scales (TES). Contemporary Educational Psychology, 46, 148-163. DOI: $10.1016 /$ j.cedpsych.2016.05.003

Garritz, A. (2010). La enseñanza de la ciencia en una sociedad con incertidumbre y cambios acelerados. Enseñanza de las Ciencias, 28(3), 315-326.

Garritz, A., Nieto, E., Padilla, K., Reyes, F. y Trinidad, R. (2015). Conocimiento didáctico del contenido en química. Lo que todo profesor debería poseer. Campo Abierto. Revista De Educación, 27(1), 153-177.

Gess-Newsome, J. (2015). A model of teacher professional knowledge and skill including PCK: Results of the thinking from the PCK Summit. En A. Berry, P. Friedrichsen y J. Loughran (Eds.), Re-examining Pedagogical Content Knowledge in Science Education (pp. 28-42). Routledge. 
Gess-Newsome, J., Taylor, J. A., Carlson, J., Gardner, A. L., Wilson, C. D. y Stuhlsatz, M. A. M. (2017). Teacher pedagogical content knowledge, practice, and student achievement. International Journal of Science Education, 1-20. DOI: 10.1080/09500693.2016.1265158

Greene, J. C. (2008). Is mixed methods social inquiry a distinctive methodology? Journal of Mixed Methods Research, 2(1), 7-22. DOI: 10.1177/1558689807309969

Hagenauer, G. y Volet, S. (2014). 'I don't think I could, you know, just teach without any emotion': exploring the nature and origin of university teachers' emotions. Research Papers in Education, 29(2), 240-262. DOI: 10.1080/02671522.2012.754929

Hagenauer, G., Hascher, T. y Volet, S. (2015). Teacher emotions in the classroom: associations with students' engagement, classroom discipline and the interpersonal teacher-student relationship. European Journal of Psychology of Education, 30(4), 385-403. DOI: 10.2307/24763247

Hargreaves, A. (1998). The emotional practice of teaching. Teaching and Teacher Education, 14(8), 835-854. DOI: $10.1016 /$ S0742-051X (98)00025-0

Hargreaves, A. (2000). Mixed emotions: teachers' perceptions of their interactions with students. Teaching and Teacher Education, 16(8), 811-826. DOI: 10.1016/S0742-051X(00)00028-7

Imbernón, F. (2012). La investigación sobre y con el profesorado. La repercusión en la formación del profesorado. ¿cómo se investiga? Revista Electrónica de Investigación Educativa, 14(2), 1-9.

Jeong, J. S., González-Gómez, D. y Cañada-Cañada (2016). Students' Perceptions and Emotions Toward Learning in a Flipped General Science Classroom. Journal of Science Education and Technology, 25, 747758. DOI: $10.1007 / \mathrm{s} 10956-016-9630-8$

Jiménez-Pérez, R., y Wamba, A. M. (2004). ¿Podemos construir un modelo de profesor que sirva de referencia para la formación de profesores en didáctica de las ciencias experimentales? Profesorado, Revista de Currículum y Formación del profesorado, 8(1), 1-16.

Kirschner, S., Borowski, A., Fischer, H. E., Gess-Newsome, J. y von Aufschnaiter, C. (2016). Developing and evaluating a paper-and-pencil test to assess components of physics teachers' pedagogical content knowledge. International Journal of Science Education, 38(8), 1343-1372. DOI: 10.1080/09500693.2016.1190479

Marcos-Merino, J. M., Esteban, R. y Ochoa, J. (2020). Valor subjetivo y emociones hacia el uso de Química en una práctica activa interdisciplinar. Educación Quimica, 31(4), 101-111. DOI: 10.22201 /fq.18708404e.2020.4.76221

Matas, A. (2018). Diseño del formato de escalas tipo Likert: un estado de la cuestión. Revista Electrónica de Investigación Educativa, 20(1), 38-47.

Mellado, L., de la Montaña, J. L., Luengo, Mar. R. y Bermejo, Mª L. (2017a). Cambios en las emociones y en las metáforas sobre el rol docente y del alumnado, del futuro profesorado de Ciencias de Secundaria, tras las prácticas de enseñanza. Revista Eureka sobre Enseñanza y Divulgación de las Ciencias, 14(2), 487-504.

Mellado, L., de la Montaña, J. L., Luengo, Ma . R. y Bermejo, Mª L. (2017b). Las emociones en las metáforas personales de futuros profesores de Secundaria en relación con el rol del profesor y del alumnado. Investigación en la Escuela, 91, 36-55.

Mellado, V., Borrachero, A. B., Brígido, M., Melo, L. V., Dávila-Acedo, Mª. A., Cañada, F., Conde, M. C., Costillo, E., Cubero, J., Esteban, R., Martínez, G., Ruiz, C., Sánchez, J., Garritz, A., Mellado, L., VázquezBernal, B., Jiménez-Pérez, R. y Bermejo, M. L. (2014). Las emociones en la enseñanza de las ciencias. Enseñanza de las Ciencias, 32(3), 11-36. DOI: 10.5565/rev/ensciencias.1478

Melo, L. V., Cañada, F. y Mellado, V. (2017). Exploring the emotions in Pedagogical Content Knowledge about the electric field. International Journal of Science Education, 39(8), 1025-1044. DOI: 10.1080/09500693.2017.1313467

Ministerio de Educación, Cultura y Deporte (2014). Real Decreto 126/2014, de 28 de febrero, por el que se establece el currículo básico de la Educación Primaria. BOE, 52, 19349-19420.

Morris, J. A. y Feldman, D. C. (1996). The dimensions, antecedents, and consequences of emotional labor. Academy of Management Review, 21, 986-1010. DOI: 10.2307/259161

Newberry, M. (2010). Identified phases in the building and maintaining of positive teacher-student relationships. Teaching and Teacher Education, 26(8), 1695-1703. DOI: 10.1016/j.tate.2010.06.022

Retana-Alvarado, D. A., de las Heras-Pérez, M. Á., Jiménez-Pérez, R. y Vázquez-Bernal, B. (2017). Emociones de maestros en formación inicial sobre la Didáctica de las Ciencias antes de una intervención indagatoria. Enseñanza de las Ciencias, n. ${ }^{\circ}$ extraordinario, 5415-5421.

Retana-Alvarado, D. A., de las Heras-Pérez, M. Á., Vázquez-Bernal, B. y Jiménez-Pérez, R. (2018). El cambio en las emociones de maestros en formación inicial hacia el clima de aula en una intervención basada en investigación escolar. Revista Eureka sobre Enseñanza y Divulgación de las Ciencias, 15(2), 2602-2618. DOI: 10.25267/Rev_Eureka_ensen_divulg_cienc.2018.v15.i2.2602 
Retana-Alvarado, D. A., de las Heras-Pérez, M. Á., Vázquez-Bernal, B. y Jiménez-Pérez, R. (2019a). ¿Cómo cambian las emociones en docentes en formación inicial al participar en un proyecto de indagación de aula? Ápice. Revista de Educación Cientifica, 3(2), 55-69. DOI: 10.17979/arec.2019.3.2.4629

Retana-Alvarado, D. A., de las Heras-Pérez, M. Á., Vázquez-Bernal, B. y Jiménez-Pérez, R. (2019b). Emociones de maestros en formación inicial hacia los contenidos científicos según el género al comienzo de una asignatura de Didáctica de Ciencias Experimentales. Revista Biografía. Escritos sobre la Biología y su enseñanza, número extraordinario, 683-695.

Rodríguez, G., Gil, J. y García, E. (1996). Metodología de la investigación cualitativa. Ediciones Aljibe.

Schwarzer, C. y Schreiber-Neumann, W. (1990). Teacher Enthusiasm: Congruence between Ratings and Behaviour. En J. M. Pieters, K. Breuer y P. R. J. Simons (Eds.), Learning Environments. Recent Research in Psychology (pp. 55-65). Springer.

Shapiro, S. (2010). Revisiting the teachers' lounge: Reflections on emotional experience and teacher identity. Teaching and Teacher Education, 26, 616-621. DOI: 10.1016/j.tate.2009.09.009

Sutton, R. E. y Harper, E. (2009). Teachers' Emotion Regulation. En L. J. Saha y A. G. Dworkin (Eds.), International Handbook of Research on Teachers and Teaching (pp. 389-402). Springer.

Sutton, R. y Wheathley, K. (2003). Teachers' emotions and teaching: A review of the literature and directions for the future research. Educational Psychology Review, 15, 327-358. DOI: 10.1023/A:1026131715856

Treagust, D., Won, M., y Duit, R. (2014). Paradigms in Science Education Research. En N. G. Lederman y S. Abell (Eds.), Handbook of Research on Science Education (Vol. II) (pp. 3-17). Routledge.

Uitto, M., Jokikokko, K. y Estola, E. (2015). Virtual special issue on teachers and emotions in Teaching and teacher education (TATE) in 1985-2014. Teaching and Teacher Education, 50, 124-135. DOI: 10.1016/j.tate.2015.05.008

van Driel, J. H., Berry, A. y Meirink, J. (2014). Research on Science Teacher Knowledge. En N. G. Lederman y S. Abell (Eds.), Handbook of Research on Science Education (Vol. 2) (pp. 848-870). Routledge.

Vázquez-Bernal, B., Jiménez-Pérez, R. y Mellado, V. (2007). El desarrollo profesional del profesorado de ciencias como integración reflexión y práctica. La Hipótesis de la Complejidad. Revista Eureka sobre Enseñanza y Divulgación de las Ciencias, 4(3), 372-393.

Vázquez-Bernal, B., Jiménez-Pérez, R., y Mellado, V. (2008). ¿Cómo podemos llevar a cabo una investigación-acción para mejorar la práctica en el aula de ciencias? Investigações em Ensino de Ciências, 13(1), 45-64.

Vázquez-Bernal, B., Jiménez-Pérez, R. y Mellado, V. (2010). Los obstáculos para el desarrollo profesional de una profesora de enseñanza secundaria en ciencias experimentales. Estudio de casos. Enseñanza de las Ciencias, 28(3), 417-432.

Vázquez-Bernal, B., Mellado, V., Jiménez-Pérez, R., y Taboada, M. C. (2012). The process of change in a science teacher's professional development: A case study based on the types of problems in the classroom. Science Education, 96(2), 337-363. DOI: 10.1002/sce.20474

Vázquez-Bernal, B., Jiménez-Pérez, R., y Mellado, V. (2013). Pedagogical Content Knowledge and ActionResearch: a Shared Strategy for Professional Development. En Y. Bashevis and Y. Weidenseld (Eds.) Professional Development: Perspectives, Strategies and Practices (pp. 1-36). Nova Science Publishers, Inc.

Zembylas, M. (2003). Emotions and Teacher Identity; a poststructural perspective. Teacher and Teaching: theory and practice, 9(3), 213-238. DOI: 10.1080/13540600309378 


\section{Anexo I. Cuestionario de emociones hacia la formadora}

Este cuestionario forma parte de una investigación cuyo objetivo es mejorar el proceso de enseñanza y aprendizaje. Completa con bolígrafo de tinta azul o negra y responde con la mayor sinceridad. Asegúrate que has respondido todas las preguntas. Muchas gracias por tu colaboración.

I Parte. Información general del estudiante. Responde los ítems marcando una equis (X) dentro del paréntesis correspondiente o indica la respuesta en el espacio que se te proporciona.

1. Sexo: ( ) Hombre ( ) Mujer

2. Edad:

3. Últimos cuatro números de tu carné universitario:

4. Grupo en el cual estás matriculado(a):

5. Acceso a la universidad: ( ) Bachillerato (pasa al ítem 6 )

( ) Formación Profesional (pasa al ítem 7)

( ) Prueba $>25$ años

6. Modalidad de Bachillerato:

( ) Artes ( ) Ciencias de la Naturaleza y la Salud ( ) Tecnología ( ) Humanidades ( )

Ciencias Sociales

\section{Modalidad de Formación Profesional:}

\section{Parte. Emociones de estudiantes del Grado en Maestro/a de Educación Primaria.}

Lee la lista de emociones que se exponen a continuación y consulta el glosario de emociones. Luego rodea en un círculo el número que mejor describa la emoción que sientes frente a la formadora. Asegúrate de marcar solamente un número para cada emoción. Los números están organizados según el grado de acuerdo de las emociones que manifiestas.

1. ¿Qué emociones sientes en relación con la formadora de la asignatura?

\begin{tabular}{cccccc}
\hline Emociones & $\begin{array}{c}\text { Totalmente } \\
\text { en } \\
\text { desacuerdo }\end{array}$ & $\begin{array}{c}\text { En } \\
\text { desacuerd } \\
\text { o }\end{array}$ & $\begin{array}{c}\text { Ni de acuerdo } \\
\text { ni en } \\
\text { desacuerdo }\end{array}$ & $\begin{array}{c}\text { De } \\
\text { acuerdo }\end{array}$ & $\begin{array}{c}\text { Totalmente } \\
\text { de acuerdo }\end{array}$ \\
\hline Susto & 1 & 2 & 3 & 4 & 5 \\
\hline Entusiasmo & 1 & 2 & 3 & 4 & 5 \\
\hline Temor & 1 & 2 & 3 & 4 & 5 \\
\hline Satisfacción & 1 & 2 & 3 & 4 & 5 \\
\hline Enojo & 1 & 2 & 3 & 4 & 5 \\
\hline Interés & 1 & 2 & 3 & 4 & 5 \\
\hline Tensión & 1 & 2 & 3 & 4 & 5 \\
\hline Aceptación & 1 & 2 & 3 & 4 & 5 \\
\hline Frustración & 1 & 2 & 3 & 4 & 5 \\
\hline Tranquilidad & 1 & 2 & 3 & 4 & 5 \\
\hline Aburrimiento & 1 & 2 & 3 & 4 & 5 \\
\hline Bienestar & 1 & 2 & 3 & 4 & 5 \\
\hline Rechazo & 1 & 2 & 3 & 4 & 5 \\
\hline Asombro & 1 & 2 & 3 & 4 & 5 \\
\hline
\end{tabular}

